\title{
Verhalten des Morphins beim reduzierenden Abbau nach Emde
}

\author{
Von \\ Franz Faltis und Theodor Heczko \\ Aus dem Chemischen Institut der Universität Graz
}

(Vorgelegt in der Sitzung am 4. Mai 1922)

Die von H. Emde ${ }^{1}$ in die organische Methodik eingeführte Spaltung quartärer Ammoniumchloride in tertiäre Basen mit Natriumamalgam, die ihre Hauptbedeutung in der Aufspaltung zyklischer Basen als Ergänzung des Hofmann'schen Abbaues beim Studium der Alkaloide besitzt, ist dann von J. v. Braun im Zusammenhang mit seinen weitreichenden Untersuchungen über die Ringfestigkeit zyklischer Stickstoffverbindungen eingehender untersucht worden. Sie wurde zuerst von M. Scholt $z^{2}$ und dann dem einen von uns ${ }^{3}$ beim Abbau eines Alkaloids, des Isochondodendrins, angewendet und später von letzterem ${ }^{4}$ auf eine andere Benzyltetrahydroisochinolinbase übertragen worden, nämlich auf das Apomorphin. Jetzt soll über die Anwendung dieser Reaktion auf die Muttersubstanz des Apomorphins berichtet werden, auf das Morphin selbst mit bedeutend komplizierterem Bau, das im Gegensatz zu den bis jetzt untersuchten Alkaloiden nur einen aromatischen Kern enthält.

Es ist vielleicht nicht überflüssig, zuerst einen Überblick über den Anwendungsbereich dieser Methode zu geben. Sie ist, soweit die Untersuchungen reichen, nur dann mit Erfolg zu gebrauchen, wenn in der Nähe des Stickstoffes sich ungesättigte Gruppen im Molekül befinden, die einen lockernden Einfluß auf die Bindung $C-N$ ausüben, sodaß Emde die Spaltbarkeit mit Natriumamalgam

\footnotetext{
1 C., 1910, II, 1477; Ar., 391, 88 (1912).

2 Ar., 252, 513 (1914).

3 Faltis und Neumann, M 42, 327 (1921).

4 Faltis und Krausz, ibid. 377 .
} 
geradezu als Maß für die Festigkeit dieser Bindung bezeichnet. Für die Öffnung vollständig hydrierter Ringsysteme, wie etwa des $N$-Dimethylpiperidiniumchlorids, als Hydroxyd das klassische Objekt des Hofmann'schen Abbaues, kommt diese Methode nicht in Betracht.

Wenn von dem bedeutenden Einfluß der Carbonylgruppe (das Carboxyl hingegen ist wirkungslos) abgesehen wird, läßt sich feststellen, daß olefinische Doppelbindung nur in unmittelbarer Nähe des Stickstoffes (in $\alpha$-Stellung) einen merklichen Einfluß ausübt, wie es in der leichten Zersetzlichkeit des Neurins unter $\mathrm{N}\left(\mathrm{CH}_{3}\right)_{3}$-Bildung zum Ausdruck kommt. In $\beta$-Stellung ist ein solcher lzaum mehr zu erkennen, da Allyltrimethylammoniumchlorid von Natriumamalgam nicht angegriffen wird. Inwieweit eine olefinische Doppelbindung im Stickstoffringsystem dieses der Aufspaltung nach Emde zugänglich macht, ist noch nicht untersucht.

Viel weiter reichend ist der Einfluß der Phenylgruppe, worüber sich ein ziemlich großes Tatsachenmaterial findet; dies sei kurz besprochen, geordnet nach der Entfernung des Phenyls vom Stickstoff in den einzelnen Fällen, wobei sich zeigen wird, daß Eingliederung des Phenyls in den Stickstoffring (in kondensierten Systemen) je nach der Natur des Ringes eigentümliche Beeinflussung der Resultate zeigt. ${ }^{1}$

\section{1. $\alpha$-Stellung des Phenyls.}

Phenyltrimethylammoniumchlorid ist mit Natriumamalgam nicht. spaltbar, was nicht überraschend ist, da die Gruppe $\mathrm{C}_{6} \mathrm{H}_{5}$ an letzter Stelle der Radikale bezüglich Beweglichkeit bei der thermischen Zersetzung quartärer Ammoniumsalze, beziehungsweise - hydroxyde (Hofmann'scher Abbau) steht. ${ }^{2}$

In kondensierten Systemen hingegen zeigt sich beim Abbau nach Emde deutlich lockernder Einfluß. Am N-Methyltetrahydrochinolinmethylchlorid, das beim Hofmann'schen Abbau ausschließlich die relativ bewegliche Methylgruppe abspaltet, tritt bei dem Vertahren nach Emde zu $60 \%$ eine andere Spaltung ein. Es bildet sich unter Reduktion die Base $\mathrm{C}_{6} \mathrm{H}_{5} \cdot \mathrm{CH}_{2}-\mathrm{CH}_{2}-\mathrm{CH}_{2} \cdot \mathrm{N}\left(\mathrm{CH}_{3}\right)_{2}$. Schon von Emde (A., 1. c.) beobachtet, wurde diese Spaltung von Braun und $\mathrm{E}$. Aust ${ }^{3}$ richtig gedeutet. Sie machten auch die interessante Feststellung, daß bei Ersatz eines Methyls in dieser Verbindung durch die beweglichere Allylgruppe die Spaltung mit Natriumamalgam ausschließlich unter Abstoßung dieses Restes und der Rückbildung von Kairolin vor sich geht.

1 Vgl. die treffende Bemerkung J. v. Braun's über das rätselhafte Gesamtspiel der Kräfte in Ringsystemen. B., 49, 2631 (1916).

2 Vgl. Braun, A., 382, 3 und 5 (1911).

3 B., 49, 501 (1916). 
Julolidinmethylchlorid I gibt ähnlich zu $63 \%$ Julolidin zurück, zu $37 \%$ reduktiv die fettaromatische Base II. ${ }^{1}$ Eine lockernde Wirkung des Phenyls in der $\delta$-Stellung ist in beiden Fällen nicht zu beobachten.<smiles>CC1CCCC2CCCCC12C(Cl)(Cl)Cl</smiles>

I.<smiles>CCC1CCCC2CCCCN(C)CCC12</smiles>

II.

Bei den $N$-Methylhydroindolbasen, die ebenfalls beim Hofmannschen Abbau nur Abspaltung des Methyls zeigen, treten, und zwar je nach den Substituenten in wechselnden Mengenverhältnissen, dieselben beiden Arten der Spaltung auf, daneben eine dritte, indem sich auch der Einfluß des Phenyls in der $y$-Stellung geltend macht ${ }^{2}$ (siehe Abschnitt 3).

\section{2. $\beta$-Stellung des Phenyls.}

Benzyltrimethylammoniumchlorid wird durch Natriumamalgam glatt reduktiv in Toluol und Trimethylamin gespalten. (Auch bei der thermischen Zersetzung ist der Benzylrest beweglicher als das Methyl.)<smiles>CC1CCCCC1C(Cc1ccccc1)[N+](C)(C)C</smiles>

III.<smiles>C[B]CCC1CCCCC1CCN(C)C</smiles>

IV.

Beim Emde-Abbau des hiehergehörigen kondensierten Systems, des $N$-Methyltetrahydroisochinolinmethylchlorids, macht sich wie beim Hofmann'schen der Einfluß der $\gamma$-Stellung überragend

1 Braun, K. Heider und W. Wyczatkowska, B., 51, 1215 (1918).

2 Braun und L. Neumann, B., 49, 1283 (1916). 
geltend (siehe Abschnitt 3) und es entsteht in beiden Fällen ausschlieblich o-Vinylbenzyldimethylamin. Dieses läßt sich nach der Methylierung des Stickstoffes nach Hoimann nicht unzersetzt weiterspalten, mit Natriumamalgam hingegen gibt es. natürlich glatt o-Vinyltoluol. Bei den Chlormethylaten der Benzyltetrahydroisochinolinalkaloide III hingegen erhöht sich der lockernde Einfluß des $\beta$-ständigen Phenyls durch den eingeführten Benzylsubstituenten und so tritt beim Abbau nach Emde schon in der ersten Stufe ausschließslich die reduktive Spaltung $\mathrm{zu}$ den inaktiven Dihydromethinbasen IV ein, während beim Hofmann'schen Abbau beide Spaltungen, die zum inaktiven $\alpha$-Methin und die zweite mögliche zur aktiven $\beta$-Methinbase $z u$ beobachten sind.

\section{3. $\gamma$-Stellung des Phenyls.}

Körper dieser Art, deren quartäre Hydroxyde im allgemeinen sehr leicht nach Hofmann, schon durch Kochen der quartären Salze mit Lauge, gespalten werden, unterliegen auch dem Abbau nach Emde, aber auffallenderweise ohne Reduktion unter. Bildung desselben Spaltstückes wie beim Hofmann-Abbau. So gibt Phenäthyltrimethylammoniumchlorid Styrol, die oben (Abschnitt 2) erwähnten Dihydromethine nach Überführung ins Chlormethylat die entsprechenden o-Vinylabkömmlinge als stickstoffreie Körper. Nur beim Dihydrodimethylapomorphimethin konnte Andeutung einer reduktiven Spaltung unter teilweiser Bildung des $o$-Aethylderivates beobachtet werden.

Das Verhalten der kondensierten Systeme in dieser Hinsicht wurde bereits am Beispiel des Tetrahydroisochinolins (siehe 2. Abschnitt), das ausschließlich $o$-Vinylbenzyldimethylamin liefert, und am Dihydroindol (siehe 1. Abschnitt) besprochen, welches zum Teil analoge Aufspaltung zeigt. Nur tritt diese nach Braun's Angabe unter Reduktion ein, unter Bildung von o-Aethylphenyldimethylamin. Doch erwähnt Braun außer den stimmenden Analysen keine Beweise für den gesättigten Charakter dieser Produkte und es wäre daher bei dem Umstand, daß die normalen Analysenfehler auf der Plusseite des $H$ und der Minusseite des $C$ liegen, die Möglichkeit nicht ganz ausgeschlossen, daß auch hier die Vinylderivate vorliegen. Es wäre daher sehr dankenswert, wenn Herr Prof. Braun diese Prüfung an seinen Präparaten vornähme, falls dies nicht schon geschehen, aber nicht besonders angeführt worden ist.

\section{D-Stellung des Phenylrestes.}

w-Phenylpropyltrimethylammoniumchlorid scheint der Aufspaltung mit Natriumamalgam nicht zugänglich zu sein; wenigstens spricht hiefür der Umstand, daß Braun die vollständige Herauslösung des Stickstoffs aus der tertiären Base, die er, wie im Abschnitt 1 
erwähnt, durch Emde-Abbau aus dem $N$-Methyltetrahydrochinolin erhalten hatte, mit Hilfe seiner $\mathrm{BrCN}$ - und $\mathrm{PCl}_{5}$-Methode durchgeführt hat.

Auch im kondensierten System ist, wie schon berichtet, bei der Spaltung nach Em de kein lockernder Einfluß in dieser Richtung wahrzunehmen gewesen, auch nicht beim $N$-Methyltetrahydrochinaldin, ${ }^{1}$ in dem die günstigste Bedingung für diese Art der Spaltung gegeben war.

Nur dem beim Emde-Abbau zu 10\% neben Methyllilolidin auftretenden zweiten Spaltprodukt des Methyllilolidinchlormethylates V schreibt Braun die Zusammensetzung VI zu, nimmt also reduktive Spaltung unter dem Einfluß der $\delta$-ständigen Phenylgruppe an,
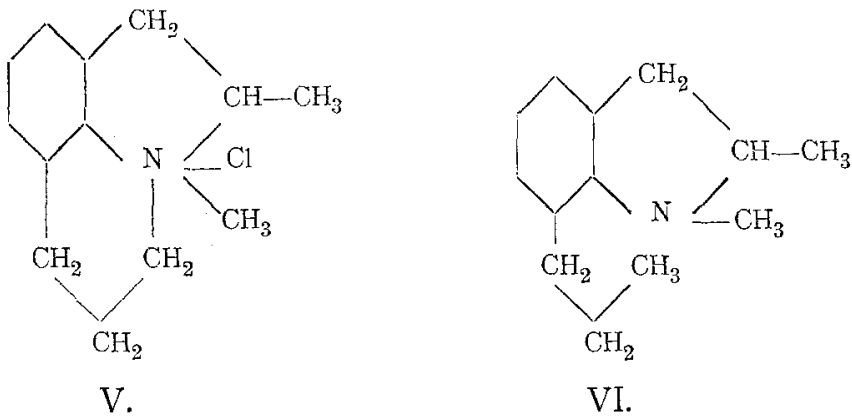

da das Produkt verschieden ist von dem von ihm für den Vergleich synthetisch dargestellten o-Propylkairolin. Da aber auch hier keine Angabe vorliegt, daß auf den gesättigten Charakter geprüft worden ist, wäre es nicht ganz von der Hand zu weisen, daß hier entsprechend dem oben angeführten Propenylkairolin vorliegt. Hiefür spräche auch, daß Lilolidinchlormethylat beim Emde-Verfahren ausschließlich Lilolidin liefert, was in Parallele damit zu setzen wäre, daß $N$-Methylhydroindolmethylchlorid bloß zu $8 \%$, das am $\alpha$-Kohlenstoff methylierte Derivat hingegen in ganz außergewöhnlichem Maße, zu $40 \%$ zur offenen aromatischen Base aufgespalten wird.

Durch diese Auffassung würde auch das Überraschende verschwinden, das für Braun die oben im Abschnitt 1 erwähnte Aufspaltung des Julolidinchlormethylates hatte, da er natürlich in Analogie zum Methyllilolidin das Entstehen von o-Propylkairolin erwartete. Unter der Annahme, daß der lockernde Einfluß der Phenylgruppe in der durch die $\delta$-Stellung gegebenen Entfernung nicht mehr zur Geltung kommt, wofür sonst alle Beobachtungen sprechen, sind die beiden oben beschriebenen Arten der Spaltung des Julolidirdimethylchlorids die zwei einzig möglichen.

1 Braun und L. Neumann, B., 50, 50 (1917). 
5. Am wirkungsvollsten hat sich der Cinnamylrest, die Kombination von Phenyl und olefinischer Doppelbindung in $\beta$-Stellung zum Stickstoff erwiesen: Cinnamyltrimethylammoniumchlorid $\mathrm{C}_{6} \mathrm{H}_{5} \mathrm{CH}=\mathrm{CHCH}_{2} \mathrm{~N}\left(\mathrm{CH}_{3}\right)_{3} \mathrm{Cl}$ sowie Cinnamylbenzyldimethylammoniumchlorid geben glatt und quantitativ unter $H$-Aufnahme Phenylpropylen. Der Cinnamylrest übertrifft also an lockernder Wirkung weitaus die Benzylgruppe. ${ }^{1}$

Es war nun von Interesse, die Muttersubstanz des bereits untersuchten Apomorphins, das:Morphin selbst, dem reduzierenden Abbau nach Emde zu unterwerfen. Beim Vergleich der Formeln für Dimethylapomorphin- (VII) und Dimethylmorphinchlormetnylat (VIII) ergibt sich, daß der im ersten Fall vorhandene Einfluß des

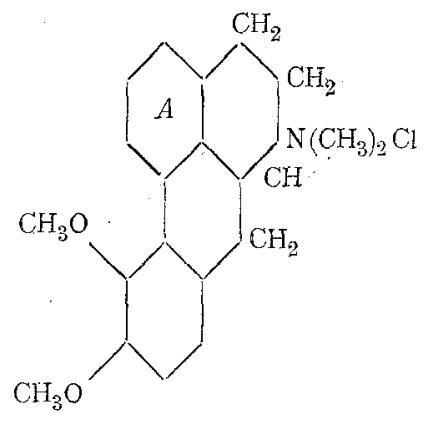

VII.

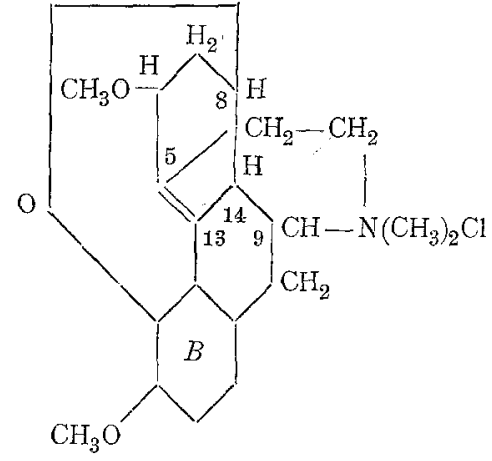

VIII. ${ }^{2}$

$\beta$-ständigen Benzolringes $A$ beim Morphin wegfäl1t, da er hier tetrahydriert ist; so kommt nur noch der $\gamma$-ständige Kern $B$, eingegliedert in den neungliederigen Heterozykel, als maßgebend in Betracht.

Das Ergebnis fiel so aus, wie es die im Abschnitt 3 besprochene Regelmäßigkeit erwarten ließ: es wurde das bereits von Pschorr aus demselben Ausgangsmaterial durch Hofmann'schen Abbau dargestellte $\alpha$-Dimethylmorphimethin erhalten. Im nichtreduktiven Verlaufe der Spaltung liegt zugleich ein Beweis für die angenommene $\gamma$-Stellung des Benzolkerns zum Stickstoff, da nur in diesem Falle beim Abbau nach Emde ein solcher bis jetzt beobachtet wurde.

Dies ist von Bedeutung im Hinblick auf die von Gadamer ${ }^{3}$ aus stereochemischen Gründen in Erwägung gezogene Morphinformel, die sich von der hier benützten dadurch unterscheidet, daß die Sauerstoffbrücke an das. $C$-Atom 5 angreift und die Haftstelle

1 Emde und H. Schellbach, Ar., 249, 118 (1911).

2 Formel nach Faltis, Ar., 255, 85 (1917).

3 Z. Ang., 26, 625 (1913). 
des Stickstoffs von $\mathrm{C}_{9}$ nach $\mathrm{C}_{8}$ verlegt, dafür die Doppelbindung $z$ wischen $\mathrm{C}_{13}$ und $\mathrm{C}_{14}$ angenommen wird. Diese Formel verdunkelt nicht nur den genetischen Zusammenhang mit dem Apomorphin, sondern ließe auch beim Emde-Abbau einen anderen Verlauf erwarten. Denn hier müßte die im Abschnitt 5 angeführte Kom: bination von Phenyl und Doppelbindung sich durch glatte Reduktion äußern und ein Dihydrodimethylmorphimethin entstehen.

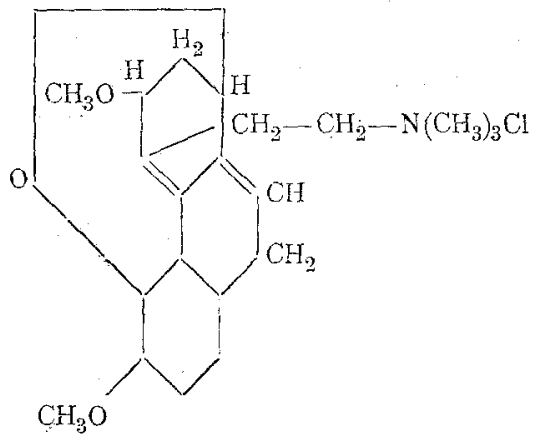

IX.

Es wurde weiter versucht, das a-Dimethylmorphimethinmethylchlorid IX dem Abbau nach Emde zu unterwerfen. Derselbe ergab ein minimales Resultat, was nicht überraschend ist, da sich die lockernde Wirkung des Benzolkernes infolge seiner großen Entfernung vom Stickstoff nicht mehr geltend macht. Ein eventueller Einfluß der in $\gamma$-Stellung befindlichen konjugierten Doppelbindung wird wahrscheinlich allmählich durch sekundäre Reduktion derselben durch den nascierenden Wasserstoff aufgehoben. ${ }^{1}$

Zum Schlusse mögen noch einige Bemerkungen über das Verhältnis des Hofmann'schen und des reduzierenden Abbaues zueinander Platz finden.

Die Richtung des thermischen Zerfalles des Systems NR1 $\mathrm{R}^{2} \mathrm{R}^{3} \mathrm{R}^{4}+\mathrm{OH}^{\prime}$ in $\mathrm{NR}^{1} \mathrm{R}^{2} \mathrm{R}^{3}$ und $\mathrm{R}^{4} \mathrm{OH}$ hängt natürlich von der Art der Substituenten ab. Zwei verschiedene Umstände machen sich hiebei in nichtzyklischen Systemen, die leichter zu überblicken sind, geltend, wie schon angedeutet: 1. Die Beweglichkeit des Restes als solchen, wobei $\mathrm{H}$ an erster Stelle steht, in großer Entfernung $\mathrm{CH}_{3}$ und daran anschließend $\mathrm{C}_{n} \mathrm{H}_{2} n+1$ mit wachsendem $n$ folgen. 2. Lockerung der Haftfestiglseit des $R$ durch in ihm enthaltene ungesättigte Gruppen, etwa in der Reihenfolge $\mathrm{CH}_{2}=\mathrm{CH}-, \mathrm{CH}_{2}=\mathrm{CH}-\mathrm{CH}_{2}-, \mathrm{C}_{6} \mathrm{H}_{5} \mathrm{CH}_{2}-,\left(\mathrm{CH}_{3}\right)$. Hiezu kommt beim thermischen Zerfall der quartären Hydroxyde (was ihn nach Braun's Untersuchur.g ${ }^{2}$ von dem der Salze unterscheidet) noch die Tendenz, beim Erhitzen Olefine von möglichst symmetrischem Bau unter Wasseraustritt $z u$ erzeugen.

I Diesbezïglich vg1. M. 42,355 und 383 , sowie die glatte Reduktion von a-Metbylmorphimethin zum Dihydroprodukt mit Na-Alkohol (E. Vongerichten, B. 32, 1047 (1899), bei der die verbleibende Doppelbindung in die $\delta$-Stellung wandern dürfte.

2 A., 382, 1 1911) 
In Ringsystemen können auch andere Ursachen das Bild verwickelic: gestalten: $\mathrm{C}_{6} \mathrm{H}_{5} \mathrm{CH}_{2}-\mathrm{CH}_{2}-$ stellt durch die lockernde Wirkung des Phenyls eir.. sehr reaktive Gruppe dar. An dem Beispiel des N-Methyltetrahydroisochinolinmethylhydroxyds wurde dies auch bestätigt gefunden, da dieses beim Hofmann'schen Abbat $O$-Vinylbenzyldimethylamin gibt. $N$-Methylhydroindolmethylhydroxyd bei derselben mabgebenden Entfernung des Phenyls vom Stickstoff setzt hingegen, wie schon angeführt, der Ringöffnung vollständigen. Widerstand entgegen.

Dies ist vielleicht darauf zurückzurühren, daß bei der Fixiertheit $\operatorname{der} \mathrm{N}\left(\mathrm{CH}_{3}\right)_{2}$ Gruppe in $z$ weiten Fall die Zerfallsreaktion $X$, wenn sie auch tiberwiegend sein sollte, reversibel geartet ist, während cer weniger weitgehende Zerfall in XI irreversibel ist und daher in seinem Ergebnis weitaus überwiegt.

Bei der Beweglichkeit des $\mathrm{N}\left(\mathrm{CH}_{3}\right)_{2}$ am Benzylkohlenstoff im ersterwähnten Beispiel ist die Spaltung in XII nur zum geringsten Teil reversibel und fïhrt daher fast ausschlieflich unter sekundärer Wasserabspaltung zum Vinylkörper als Endprodukt.<smiles>C[N+]1(C)CCC2CCCCC21</smiles><smiles>CN(C)C1CCCCC1CCO</smiles>

X.<smiles>CN1CCCCC1CO</smiles>

XI.<smiles>CN(C)CC1CCCCC1CCO</smiles>

XII.

Wenn wir uns nun dem Abbau der quartären Chloride durch Natriumamalgam zuwenden, fält vor allem auf, daf er schon bei kleiner $\mathrm{OH}^{\prime}$-Konzentration beginnt (falls er überhaupt eintritt), bei viel geringerer, als sie bei der Modifikation des Hofmannschen Abbaues angewendet werden mul, die darin besteht, daß quartäre Salze mit konzentrierter Lauge zum Sieden erhitzt werden. Das Wesen des reduzierenden Abbaties besteht vielleicht darin, dâ Natrium nicht nur auf $\mathrm{H}$ - unter Bildung von $1 / 2 \mathrm{H}_{2}$ ladungsentziehend wirkt, sondern anch mit anderen positiv geladenen Ionen, die sich in der wässerigen Lösung finden, sich in derselben Art umsetzt. Dies wird um so mehr in den Vordergrund treten, je größer ihre Konzentration in der Lösung ist:

$$
N R^{1} R^{2} R^{3} R^{4} \bullet+N a \rightarrow R^{1} R^{2} R^{3} R^{4}+N a \bullet
$$

Das vom Natrium abgegebene Elektron wird um den positiven Komplex $N^{1} R^{2} R^{3} R^{4}$ kreisen; ist eines der Radikale $H$, so wird sich das Elektron $\Theta$ mit 
diesem verbinden, da die maximale Koordinationszahl des Wasserstoffs 2 ist (vgl. die Existenz von $\left(\mathrm{NH}_{3}\right)_{2} \mathrm{HOH}$, von $\mathrm{Py}_{2} \mathrm{HCl}$ usw.). Das Gebilde

$$
\mathrm{R}_{1} \div \mathrm{N}_{5} \div \mathrm{H}_{1}-\theta
$$

(wobei - die Atomverknüpfung durch ein kreisendes Elektronenpaar andeuten und die neben den Atomzeichen stehenden Indizes die Zahl der disponibien positiven Ladungen bezeichnen soll) zerfällt aber in

$$
\begin{aligned}
& \mathrm{R}_{1} \\
& \text {-1. } \\
& \mathrm{R}_{1} \div \mathrm{N}_{5} \div+\mathrm{H}_{1}-\theta \text {, beziehungsweise } \mathrm{H}_{1} \div \mathrm{H}_{1} \text {, } \\
& \text {. } 1 \text {. } \\
& \mathrm{R}_{1}
\end{aligned}
$$

da der vierte taube Elektronenkreis zwar befähigt ist, lokal Anziehung auf ein Kation, etwa $\mathrm{H} \bullet$ auszuüben und es zu binden (Bildung des komplexen Ammoniumions), neutral gewordene Atome (oder Molekell) aber nur mehr sehr locker. gebunden hält.

Sind jedoch, wie in den quartären Salzen, alle vier $R$ Kohlenstoffreste, so ist bei dem Umstand, daß bei $\mathrm{C}$ und bei $\mathrm{N}$ (um diesen herum gruppiert sich das Überwiegen der positiven um die negativen Ladungen) die maximale Koordinationszahl 4 beträgt, die hier schon besetzt ist, eine Stabilisierung des kreisenden Elektrons in der Koordinationssphäre des $\mathrm{N}$ oder $\mathrm{C}$ nicht möglich. 2 Dieses wird sich daher mit dem überall vorhandenen $\mathrm{H} \bullet$ (des Wassers) $z u \mathrm{H}_{1}-\Theta$, beziehungsweise $\mathrm{H} \div \mathrm{H}$ verbinden. Die Reaktion verläuft also praktisch ausschlieflich unter Wasserstoffentwicklung, am Schlusse findet sich die unangegriffene quartäre Base in der Lösung.

Sind aber gelockerte C-N-Bindungen im Komplex vorhanden, so kann Verbindung des Elektrons mit dem in minimalem Maße sich vorfindenden Jon $R_{1} \bullet$ eintreten. $R_{1}-\Theta$ wird bei großer Verbindungstendenz mit $H_{1}-\Theta$ (das durch die Reaktion des Na mit Wasser ständig entsteht) sich mit diesem vereinigen unter

Bildung von $\mathrm{R}_{1} \div \mathrm{H}_{1}$. Ist diese Verbindungstendenz eine ganz besonders ausgeprägte, so können auch Zerfallsrichtungen zum Vorschein kommen, die beim thermischen Zerfall kaum angedeutet sind. So bei der Reduktion des $N$-Methyltetrahydrochinolinchlormethylates $\mathrm{zu} \omega$-Phenylpropyldimethylamin.

Nur bei $\gamma$-ständigem Phenyl tritt wegen geringer Verbindungstendenz mit Wasserstoff eine andere Art der Stabilisierung des Radikals $\mathrm{C}_{6} \mathrm{H}_{5} \mathrm{CH}_{2}-\mathrm{CH}_{2}-\Theta$ ein. Es wird $\mathrm{H}$ unter Bildung des Olefins $\mathrm{C}_{6} \mathrm{H}_{5} \mathrm{CH}=\mathrm{CH}_{2}$ abgespalten. Im Fall

1 Z. B. $\mathrm{H}_{2} \mathrm{O}$ im undissoziierten Anteil des $\mathrm{NH}_{4} \mathrm{OH}$.

2 Ersetzt man in dieser Überlegung das kreisende Elektron, das nicht in die Koordinationssphäre des $\mathrm{N}$ und des $\mathrm{C}$ eindringen kann, durch das Hydroxylion, so liegt vielleicht in dieser * Zwangsionisierung* die Erklärung für den enormen Unterschied in der wahren Dissoziationskonstante der quartären und der tertiären Amine. Vg1. diesbezüglich T. S. Moore und Th. Winmill (Soc., 101, 1635; C. 1912, II, 2045), die durch Ermittlung der Hydratationskonstante die wahre Dissoziation einiger primärer, sekundärer und tertiärer Amine bestimmt haben, welche viel kleiner ist, als im allgemeinen angenommen wird. 
des $N$-Methylhydroindolmethylchlorids wird das Eintreten des Zerfalls in XIII im wesentlichen nicht reversibel geartet sein und daher in der teilweisen Spaltung zum o-Vinyl- (nach Braun o-Aethyl-) dimethylanilin zum Ausdruck kommen (im Gegensatz zum oben besprochenen Verlauf der Spaltung nach Hofmann).

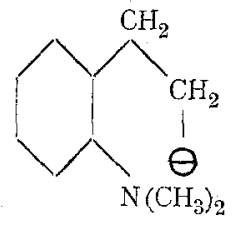

XIII.

Da es nicht ausgeschlossen war, daß das Eintreten der Spaltung quartärer Chloride mit Natriumamalgam schon bei geringer $\mathrm{OH}^{\prime}$-Konzentration auf katalytische Wirkung des $\mathrm{Hg}$ zurückzuführen ist, wurde mit Herrn Dr. Georg Watzinger diese Frage experimentell geprüft, indem $7 \mathrm{~g}$ Kairolinjodmethylat nach Überführung ins Chlormethylat mit 4 Äquivalenten $\mathrm{NaOH}$ in $50 \mathrm{~cm}^{3}$ wässeriger Lösung 8 Stunden lang am Wasserbad erwärmt wurden, und zwar zur Hälfte unter Zusatz von metallischem, zur Hälfte unter Zugabe von $1 g$ feinstverteiltem Quecksilber (erhalten durch Reduktion von $\mathrm{HgCl}_{2}$ mit $\mathrm{H}_{2} \mathrm{O}_{2}$ in alkalischer Lösung). Hiebei fanden wir nur minimale Bildung von Kairolin, nicht größer wie bei einem Kontrollversuch ohne Hg-Zusatz. ${ }^{1}$

\section{Experimenteller Teil.}

Die vollständige Methylierung des Morphins an beiden Hydroxylen und am Stickstoff wurde nach Pschorr ${ }^{2}$ mittels Dimethylsulfat und wässeriger Lauge in der Kälte durchgeführt. Nur ein kleiner Teil des gebildeten quartären Salzes wurde mit $J K$ als Jodid gefällt, um es zu identifizieren und der Analyse zu unterwerfen:

$0.2045 \mathrm{~g}$ Substanz bei $110^{\circ}$ getr. gaben bei der Methoxylbestimmung $0.2072 \mathrm{~g}$ AgJ.

Gef. $13 \cdot 95 \%$,

ber. für $\mathrm{C}_{20} \mathrm{H}_{26} \mathrm{O}_{3} \mathrm{NJ} 13 \cdot 63 \%_{0} \mathrm{OCH}_{3}$.

$[\alpha]_{D}^{16}=-109 \cdot 7^{\circ}$ in $\mathrm{H}_{2} \mathrm{O}\left(\alpha=-1 \cdot 087^{\circ}, c=0.992, l=1 \mathrm{dm}\right)$.

Pschorr fand $[\alpha]_{D}=-107 \cdot 2^{\circ}$.

Die Hauptmenge der Reaktionsflüssigkeit wurde mit Salzsäure neutralisiert, mit konzentrierter $\mathrm{HgCl}_{2}$-Lösung im Überschuß versetzt und einen Tag lang sich selbst überlassen, da das frisch

1 Vgl. Braun, B., 49, 505 (1916), der denselben Versuch ohne Hg-Zusatz ebenfalls mit negativem Erfolg durchgefüht hat.

2 B., 44, 2635 (1911). 
gefällte Quecksilberdoppelsalz relativ leicht löslich ist. Dann wurde der Niederschlag abgesaugt und mit verdünnter Sublimatlösung bis zum Verschwinden der Schwefelsäurereaktion nachgewaschen. Das lufttrockene, grauweiße Doppelsalz wurde zerrieben, in Wasser suspendiert und durch Einleiten von $\mathrm{H}_{2} \mathrm{~S}$ zersetzt. Nach dem Filtrieren von $\mathrm{HgS}$ werden das salzsaure Filtrat, welches das Methylcodeinmethylchlorid enthält, und die gesammelten Waschwässer am Wasserbad weitgehend eingeengt, und 2 war auf etwa $75 \mathrm{~cm}^{3}$ für 0.05 Grammäquivalente $(18 \mathrm{~g})$, mit $\mathrm{NaOH}$ eben alkalisch gemacht und mit Natriumamalgam zersetzt. Die Operation dauert bei Anwendung von zirka $200 \mathrm{~g} 5$ prozentigem Na-Amalgam 4 bis Stunden. Nach kurzer Zeit färbt sich die Flüssigkeit dunkel und scheidet sich an der Oberfläche das Reaktionsprodukt als Öl ab. Dabei tritt Amingeruch auf, ein Zeichen, daß die Reaktion zum Teil weitergeht unter Bildung stickstoffreier Körper. Nach dem Erkalten wird das erstarrte Öl isoliert, gut mit Wasser gewaschen, die alkalische Mutterlauge mit der gleichen Menge Äther ausgeschüttelt, in demselben Äther das isolierte Reaktionsprodukt zur Lösung gebracht und durch Ausschütteln mit verdünnter Salzsäure die Methinbase von den stickstoffreien Anteilen getrennt. Letztere hinterblieben nach dem Verjagen des Äthers in kleiner Menge als braunes Ö1. Die wässerige salzsaure Lösung wurde mit $\mathrm{NH}_{3}$ alkalisch gemacht und die ausfallende Methinbase sofort in Äther aufgenommen. Nach dem Trocknen mit Natriumsulfat wurde der Äther verjagt; es blieb ein Sirup zurück, der bald durchkrystallisierte und zur Weglösung von klebrigen Verunreinigungen mit wenig reinem Äther übergossen wurde. Nach kurzem Umschwenken wurde abgegossen und einmal mit wenig frischem Äther nachgespült. Es hinterblieb reine krystallisierte Methinbase vom Schmelzpunlit 91 bis $92^{\circ}$ korr. Die Ausbeute betrug 60\% der Theorie.

Zur Erreichung einer schönen Krystallisation wurde einmal die noch nicht durch die Ätherbehandlung von den Verunreinigungen betreite Substanz in einer Krystallisierschale in Alkohol gelöst, die Schale aufs Eis gestellt und über die Lösung Wasserdampf geleitet. Mit zunehmendem Wassergehalt des Alkohols schieden sich daraus schöne weiße, diamantglänzende Krystalle vom selben Schmelzpunkt aus, doch war die Methode verlustreich und wurde verlassen, um so mehr, da auch reine Methinbase bei längerem Erhitzen mit Alkohol eine geringe Veränderung zu erleiden scheint. Die nach dem Abdunsten des Alkohols zurückgewonnene Substanz war klebrig geworden und zeigte unschärferen Schmelzpunkt. Auch tritt dann an einer kalt gesättigten Lösung beim Erhitzen eine Entmischungserscheinung auf, welche die reine Substanz nicht zeigt und von dem Produkt einer beginnenden Zersetzung geliefert werden dürfte.

Die Methinbase erwies sich als identisch mit der von Pschorr (l. c.) aus demselben Ausgangsmaterial durch Hofmann'schen Abbau 
dargestellten a-Dimethylmorphimethin, das zum Vergleich nach seiner Vorschrift hergestellt und durch Umkrystallisieren aus Methylalkohol unter Wasserzusatz gereinigt wurde. Der Schmelzpunkt lag bei 90 bis $91^{\circ}$ korr., während Pschorr $94^{\circ}$ angibt. Der Mischschmelzpunkt mit unserer nach dem Emde-Verfahren gewonnenen Methinbase zeigte keine Depression: er lag bei 90 bis $91.5^{\circ}$.

$3.610 \mathrm{mg}$ unserer Methinbase, im Vakuum zur Gewichtskonstanz gebracht, gaben bei $20^{\circ}$ und $743 \mathrm{~mm} \mathrm{Hg} 0: 138 \mathrm{~cm}^{3} \mathrm{~N}$ (Dr. Rollett).

Gef. $4 \cdot 35 \%$,

ber. für $\mathrm{C}_{20} \mathrm{H}_{25} \mathrm{O}_{3} \mathrm{~N} 4 \cdot 28 \%$ N.

Die Bestimmung des Drehvermögens unserer Substanz ergab:

$[\alpha]_{D}^{17}=-254 \cdot 2^{\circ}$ in Methylalkohol $\left(\alpha=-1 \cdot 23^{\circ}, c=0 \cdot 484, l=1 \mathrm{dm}\right)$.

$[\alpha]_{D}^{18}=-291.2$ in 92 prozentigem Aethylalkohol $\left(\alpha=-1 \cdot 42^{\circ}, c=0.4876\right)$.

Pschorr gibt für seine Substanz in Methylalkohol $[a]_{D}=-251 \cdot 9^{\circ}$ an.

Beide Substanzen gaben die gleiche charakteristische Farbenreaktion mit konzentrierter $\mathrm{H}_{2} \mathrm{SO}_{4}$, welche jener der Methylmorphimethine ähnelt und von Pschorr nicht beschrieben ist: Eine Spur Substanz auf ein Uhrglas gebracht und mit einem Tropfen Schwefelsäure versetzt, färbt dieselbe gelbbraun, nach wenigen Sekunden rotbraun. Nach einiger Zeit geht die Farbe in Rot über und vom Rand des Tropfens treten blaue Schlieren auf. Bei längerem Stehen oder rasch durch Zusatz von einem Tropfen Wasser (Wärmewirkung) geht die Färbung über Lila in ein schönes Blau über, das bei stärkerem Verdünnen mit Wasser verschwindet, um bei abermaligem Schwefelsäurezusatz wiederzukehren. (Das Dihydromethylmorphimethin löst sich in konzentrierter $\mathrm{H}_{2} \mathrm{SO}_{4}$ mit brauner Farbe, ohne den charakteristischen Übergang in Blau zu zeigen. E. Vongerichten, 1. c.) Auch das Jodmethylat gibt beim Erwärmen mit Schwefelsäure die blaue Farbenreaktion. Sogar am ungereinigten stickstoffreien Produkt, das daraus durch Hofmann'schen Abbau genommen wurde, war sie in ähnlicher Weise zu beobachten.

Zur Vornahme des reduzierenden Abbaues in der zweiten Stufe wurde unser Dimethylmorphimethin in das Jodmethylat übergeführt. Die Base wurde in etwa der achtfachen Menge Methylalkohol gelöst und unter Rückflußkühlung mit etwas mehr als der berechneten Menge Jodmethyl zum Sieden erhitzt. Nach etwa 20 Minuten ist die ganze Masse ein Krystallbrei und die Reaktion quantitativ vor sich gegangen. Beim Umkrystallisieren aus Wasser wird die Substanz, die mit dem von Pschorr beschriebenen Jodmethylat identisch ist, in glänzenden Nadeln erhalten. daten:

Das $\alpha$-Dimethylmorphimethinmethyljodid gab folgende Analysen- 
1. $4.197 \mathrm{mg}$ Substanz bei $120^{\circ}$ getrocknet gaben $8 \cdot 185 \mathrm{mg} \mathrm{CO}_{2}$ und $2 \cdot 330 \mathrm{mg}_{\mathrm{g}} \mathrm{H}_{2} \mathrm{O}$ (Dr. Rollett).

2. 9.282 $\mathrm{mg}$ Substanz gaben bei $734 \mathrm{~mm} \mathrm{Hg}$ und $22^{\circ} 0.252 \mathrm{~cm}^{3} \mathrm{~N}$ (Dr. Wintersteiner).

3. $7.940 \mathrm{mg}$ Substanz gaben bei demselben Druck und $23^{\circ} 0.216 \mathrm{~cm}^{3} \mathrm{~N}$ (Dr. Wintersteiner.)

4. $0 \cdot 3082 g$ Substanz gaben durch Ausfällung des $\mathrm{J}$ mit $\mathrm{AgNO}_{3}$ aus der wässerigen Lösung $0.1529 \mathrm{~g}$ AgJ.

5. $0.2444 \mathrm{~g}$ Substanz gaben bei der Methoxylbestimmung $0.2330 \mathrm{~g}$ AgJ, bei der Methylimidbestimmung $0.3233 \mathrm{~g}$, bei der Wiederholung $0.0448 \mathrm{~g}$, beziehungsweise $0.0086 \mathrm{~g}$, insgesammt $0.3767 \mathrm{~g} \mathrm{AgJ}$.

Gef. 1. $53.20 \% \mathrm{C}, 6.21 \% \mathrm{H} ; 2.3 .03 \% ; 3.3 .03 \% \mathrm{~N} ; 4.26 .93 \% \mathrm{~J}$, 5. $6.10 \% \mathrm{CH}_{3}$ an $\mathrm{O}, 9.86 \% \mathrm{CH}_{3}$ an $\mathrm{N}$;

ber. fuir $\mathrm{C}_{21} \mathrm{H}_{28} \mathrm{O}_{3} \mathrm{NJ} 53 \cdot 71 \% \mathrm{C}, 6.01 \% \mathrm{H}, 2 \cdot 99 \% \mathrm{~N}, 27.050 \% \mathrm{~J}, 6.440_{0}$ $\mathrm{CH}_{3}$ an $\mathrm{O}$ und $9 \cdot 66 \% \mathrm{CH}_{3}$ an $\mathrm{N}$.

$[\alpha]_{D}^{17}=-150^{\circ}$ in Wasser $\left(\alpha=-0.74^{\circ}, c=0.493, l=1 \mathrm{dm}\right)$.

$[\alpha]_{D}^{19}=-196.3$ in Äthylalkohol + Wasser $D_{19}=0.9315 \quad\left(\alpha=-2 \cdot 01^{\circ}\right.$, $c=1 \cdot 0236)$.

Pschorr gibt für sein Jođmethylat in Wasser $[\alpha]_{D}^{23}=-134 \cdot 4^{\circ}$ an.

$7 \cdot 5 \mathrm{~g}$ Jodmethylat wurden mit $\mathrm{AgCl}$ ins Chlormethylat umgewandelt und die wässerige Lösung desselben auf $30 \mathrm{~cm}^{3}$ eingeengt. Die Reaktion, die mit $80 \mathrm{~g} 5$ prozentigem Natriumamalgam durchgeführt wurde, verlief unter mäßiger Trimethylaminentwicklung. Die braungefärbte Reaktionsflüssigkeit wurde ausgeäthert, die ätherische Schicht mit verdünnter Salzsäure versetzt, wobei vorübergehend eine starke Trübung vom Chlorhydrat der unzersetzten Base herrührend entstand, und erschöpfend ausgeschüttelt. Die braungefärbte, blau fluoreszierende ätherische Schicht wurde abgetrennt und mit $\mathrm{Na}_{2} \mathrm{SO}_{4}$ getrocknet. Nach dem Abdestillieren des Äthers hinterblieben zirka $0.1 \mathrm{~g}$ einer braunen amorphen Substanz. Damit war der Beweis erbracht, daß der reduzierende Abbau des a-Dimethylmorphimethinmethylchlorids nur spurenweise vor sich geht.

Diese Arbeit wurde durch die Unterstützung eines in Holland weilenden Landsmannes, der ungenannt bleiben will, gefördert und es sei ihm auch an dieser Stelle hiefür der wärmste Dank ausgesprochen. 